Acta Poetica $33 \cdot 2$

JULIO-DICIEMBRE

$2012(151-165)$

\title{
Kälacakra: breves consideraciones en torno del tiempo
}

\author{
Adrián Muñoz
}

Este artículo propone una lectura comparativa entre la concepción budista del tiempo y una discusión fenomenológica occidental. Centrándose sobre todo en la escuela Madhyamaka, el texto discute cómo la cuestión de la fugacidad y la impermanencia del tiempo resulta afín a las reflexiones que Gaston Bachelard elabora en su célebre La intuición del instante. En ambos casos, lo que se destaca es el énfasis en que la duración no es sino una construcción carente de una verdadera realidad absoluta, al mismo tiempo que el ser humano posee anhelos de trascendencia, los cuales, a su vez, engendran engaño y decepción.

Palabras Clave: tiempo, budismo, Gaston Bachelard, impermanencia, ciclicidad.

This paper calls for a comparative reading of both the Buddhist conception of time and a branch of Western phenomenology. With special emphasis on the Madhyamaka Buddhist school, it discusses the way in which fugacity and impermanence are similarly discussed by French philosopher Gaston Bachelard in his L'intuition de l'instant. In both cases, the stress lies on the fact that duration is but a mental construct lacking true, absolute reality, while at the same time the human being is subjected to hopes of transcendence which, in turn, engender disillusion and delusion.

KeYwords: time, Buddhism, Gaston Bachelard, impermanence, cyclic time. 



\author{
Adrián Muñoz \\ Facultad de Filosofia y Letras \\ Universidad Nacional Autónoma de México
}

\title{
Kälacakra: breves consideraciones en torno del tiempo ${ }^{1}$
}

El tiempo es la substancia de que estoy hecho.

El tiempo es un río que me arrebata, pero yo soy el río; es un tigre que me destroza, pero yo soy el tigre; es un fuego que me consume, pero yo soy el fuego.

Borges

El tiempo puede ser un grillete. En cada aspecto de la vida humana, el influjo del tiempo es determinante. Todo está pensado en función de la temporalidad, ya sea mediante referentes al pasado o con miras hacia el futuro. Esto implica ya de entrada cierta problemática que ha estado presente en el imaginario humano desde épocas inmemoriales. Las nociones de una mítica época dorada encarnan una entrañable nostalgia por un pasado en teoría moralmente más puro y bello, mientras que esto

${ }^{1}$ En cuanto al título de estas páginas, tengo en mente el comentario de Borges respecto a su "Nueva refutación del tiempo". El primer adjetivo introduce la noción de un atributo temporal, que es precisamente el punto que se va a desmenuzar en estas páginas. Además, el uso de "en torno de" agrega la noción de espacio y, así, la relación inherente entre el tiempo y el espacio (curiosamente, el título también evoca al artículo de Luis Gómez que figura en la bibliografía). 
sugiere que, por ende, el presente constituye una corrupción de la condición ideal de aquellos tiempos. En más de una tradición se habla de la pérdida de una Era Ideal y el imposible, mas añorado, retorno a ella.

En cuanto al futuro, las posiciones pueden ser más encontradas. A modo de esquema general - mas consciente de la generalización arbitraria - en el mundo europeo la historicidad de la humanidad se ha concebido en términos de un supuesto "progreso", entendido como un impulso natural de todo ser humano. De este modo, personalidades como Max Weber, Hegel o Karl Marx han supuesto que el hombre, por naturaleza, tiende hacia un perfeccionamiento y cada uno de ellos expuso sus propios parámetros. Sin embargo, respecto a Oriente (o el mundo no europeo), se desarrolló al mismo tiempo una jerarquización en cuanto al grado de desarrollo histórico. Así, partiendo de que el modelo estaba epitomado por el hombre europeo moderno, todo pueblo ajeno a dicho ideal terminó considerado como "atrasado", "bárbaro", "en vías de desarrollo", etc. El epítome del Hombre —o del espíritu humano en términos de Hegel- resultaba ser el alemán protestante moderno. Técnicas de producción, pensamiento mágico/racional, tradición versus tecnología, se convirtieron no solo en criterios para evaluar el grado de desarrollo de un pueblo en cuestión, sino que también han proporcionado numerosos debates en torno de estos y otros tópicos más generales.

¿Quién dice quién es más avanzado o no lo es? ¿Por qué los criterios deberían ser necesariamente el potencial tecnológico-científico, el nivel de "modernidad", o el potencial económico? Progreso, en su sentido original, no denota en absoluto las nociones de ciencia o tecnología, ni de florecimiento económico, sino de un proceso ascendente o, si se prefiere, en línea recta. En todo caso, ¿por qué progresar? De hecho, el conocimiento occidental mira la historia desde un punto de vista progresista; a pesar de sus posibles tensiones internas, todos apuestan hacia un gradual perfeccionamiento del ser humano. En la India antigua, por el contrario, las teorías del tiempo referían que, antes que nada, el curso de la historia era degradatorio, es decir que la calidad moral del hombre decaía en cada era. Además, la historia no era considerada como un transcurso lineal, sino como un proceso cíclico. Todo momento, eventualmente, volvería a suceder. 
Para unos, el curso de la historia constituye un devenir lineal e irreversible; para otros, no es sino un ciclo que se repite infinitamente y el cual está dividido y subdividido en eras, las cuales marcan el declive moral y espiritual del Hombre. El término sánscrito más común para referirse al tiempo es kāla. Varios autores han intentado dar una cuenta etimológica del vocablo. Así, Kuiper (véase González Reimann, 64, n. 52) deriva la palabra de la raíz indoeuropea qwel, que indica movimiento, en especial uno de tipo circular. La misma raíz ha produci-

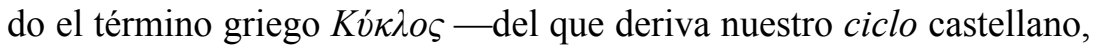
además del inglés wheel—y, significativamente, el sánscrito cakra. De modo que ya desde su primera acepción, el término kāla implica circularidad. Siendo así, no resulta extraño que eventualmente la India haya desarrollado la noción del saṃsāra, es decir la rueda o ciclo de muertes, nacimiento y reencarnaciones. Un término que es común hallar para hablar del tiempo, de hecho, es kālacakra, la gran rueda del tiempo. Aunque esto no sería sino una tautología si nos apegamos a la teoría de Kuiper.

Cuando nace esta idea del samsāra, la concepción misma del tiempo ha de sufrir alteraciones y de convertirse en objeto de debate dentro de algunas escuelas filosófico-teológicas. Dado que lo que se persigue en la India en general es la liberación de este ciclo de reencarnaciones, las diferentes corrientes han presentado numerosas vías de escape, así como han definido el tiempo de maneras diferentes. En el mundo europeo también se han suscitado numerosos debates sobre el tiempo en este sentido, más allá de las ideas progresistas a las que aludí en la primera página. En efecto, tanto en la India como en Europa diversos pensadores se han dedicado a elucidar las implicaciones de la relación entre el tiempo y el individuo o, mejor dicho, el ser. Tal vez la primera impresión nos lleva a determinar que una diferencia esencial radica en que mientras que para los pensadores occidentales el tiempo y el devenir son concebidos como fenómenos físicos y ontológicos, para los filósofos indios, grosso modo, el tiempo pertenece al ámbito de lo metafísico, en tanto trascendencia de lo material.

Estas páginas ofrecen breves apuntes sobre el tema; apenas unas notas que especulan comparativamente acerca de concepciones circulares, pero discontinuas, del tiempo. Me limitaré aquí a esbozar una o dos de 
las perspectivas con que se ha abordado este tema. No pretendo - aclaro- realizar una exposición detallada de tal o cual teoría; por el contrario, mi objetivo es más bien suscitar el diálogo entre distintas teorías $y$, aun más, leer unas en términos de otras, a fin de dar con puntos en común. Mi interés reside, en principio, en adelantar algunas consideraciones y especulaciones básicas sobre el tiempo, tal y como lo asienta el título de este texto. De esta manera, tomaré, por un lado, la posición de la escuela budista denominada Madhyamaka, en especial a partir de Nāgārjuna y su afamado texto Mūlamadhyamaka-kārikā (мМк); y, por el otro, el argumento de Gaston Bachelard presentado en La intuición del instante.

La lectura comparativa supone, curiosamente, una invitación a franquear las barreras del tiempo: Nāgārjuna escribió entre el 150 y el 250 de la era común; Bachelard, en la primera mitad del siglo xx. El anacronismo, empero, se muestra irrelevante debido a las similitudes, como espero poder señalar.

Nāgārjuna, como budista seguidor del śūnyavāda (la doctrina de la vacuidad), comienza por hacer una disolución del tiempo, el cual, concluye, no es ni una sustancia ni una realidad, y sin embargo tampoco una no-existencia absoluta; el tiempo es, antes bien, una noción derivada (Ramanan, 197). El tiempo, dice el budismo Madhyamaka, no tiene una existencia real, pero sí una convencional, lo cual se explica gracias a la teoría de la Originación Dependiente (pratītya-samutpāda), según la cual los fenómenos surgen y dependen de otros fenómenos, si bien también sus causas son el efecto o producto de otras causas o fenómenos anteriores (Arnau, 65). En otras palabras: las cosas no poseen realidad por sí mismas, sino que dependen de otras causas que, a su vez, dependen de otras y así sucesivamente. Además, los tres tiempos (pasado, presente y futuro) no pueden tener para el budismo una naturaleza intrínseca y estable porque el budismo sostiene antes que nada la impermanencia de todo objeto y toda naturaleza propia. Ciertamente, la dialéctica budista se caracteriza por ser antisustancialista, apofática y anticonceptualista 
(Gómez, "Consideraciones en torno al absoluto”, 97); ¿cómo, entonces, describir o concebir al tiempo, una de las categorías más aparentemente absolutistas que hay?

Bachelard, por su parte, se vale de la teoría de Roupnel, el otro Gaston, y hasta cierta medida de Bergson, para sostener que el tiempo solo posee una realidad única: el instante. Para desarrollar su argumentación Bachelard parte de la premisa según la cual "la filosofía bergsoniana es una filosofía de la acción; la filosofía roupneliana es una filosofía del acto" (Bachelard, 19). Esto supone que para el primero la acción es un continuo hacer que presenta la duración y la originalidad, mientras que para el segundo, el acto supone una decisión instantánea, portadora de la originalidad. Pero hay que señalar que en este caso no se trata de tomar la originalidad como una realidad vinculada con la eternalidad del ser; antes bien, tanto para Roupnel como para Bachelard el tiempo es una discontinuidad de instantes, la duración misma del tiempo está compuesta de instantes sin duración, sin demasiada relación con los instantes vecinos. La idea de permanencia se gesta no porque de hecho exista una como tal, sino por la combinación de múltiples factores:

Lo que el ser puede tener de permanente es la expresión, no de una causa inmóvil y constante, sino de una yuxtaposición de resultados fugaces e incesantes, cada uno de los cuales tiene su base solitaria y cuya ligadura, que es solo un hábito, compone a un individuo (Roupnel apud Bachelard, 21; ver también, 64).

Para Roupnel la duración no es sino una construcción que carece de realidad absoluta y que imita la permanencia mediante la memoria, la imaginación y procesos similares, es decir, por representaciones mentales. La duración y permanencia del tiempo es experimentada a causa de la repetición de instantes, del hábito.

Ahora, ¿no es algo similar lo que encontramos en las doctrinas budistas, y aun no budistas, de la India? Las escuelas que hablan de la impermanencia y la irrealidad del mundo fenoménico sugieren que la aparente realidad del mundo se debe a nuestras representaciones mentales, atrapadas y condicionadas por la contraposición de nociones, valores y conceptos; en gran medida, es un acto mental el que hace surgir al 
mundo, por así decirlo. El filósofo budista lo pone de esta manera: "La conciencia, condicionada por conformaciones mentales, se establece con respecto a las varias formas de vida. Cuando la conciencia está establecida, el nombre y la forma se hacen aparentes" (MMK, XXVI.2). ${ }^{2}$

El tiempo resulta ser únicamente una forma de medición o, mejor dicho, una tentativa de medición del devenir. Nāgārjuna se dedica a erradicar la noción de que el tiempo, y sus tres componentes, poseen una naturaleza permanente: "Aun más, no siendo contingentes con el pasado, el establecimiento del presente y futuro no se percibe. Por lo tanto, ni un tiempo presente ni uno futuro es evidente" (MMK, XIX.3). ${ }^{3}$

Se adivina aquí una tensión típica de la dialéctica budista: al tener que lidiar con la noción de un absoluto inaprehensible, o bien se recurre a una negación del fenómeno, o bien se recurre a una "reafirmación dialéctica del fenómeno después de negado" (Gómez, "Consideraciones en torno al absoluto", 108-109).

En resumidas cuentas, prosigue Nāgārjuna, todo periodo de tiempo, así como toda categoría y concepto de relación debe entenderse en el mismo sentido, es decir, carente de realidad verdadera y absoluta. Si se aplica el método de disolución ("análisis" u "ontología abolitiva" [Gómez, "Emptiness and Moral Perfection", 369]) que practica el budismo Mādhyamika, en especial en el Hastavālanāma-prakāraṇa, se verá que el presente mismo es inexistente como tal: así como una pieza de tela está compuesta de numerosas hebras y de filamentos, del mismo modo una hora no posee realidad intrínseca porque puede descomponerse en 60 minutos, y cada minuto se compone de 60 segundos; cada segundo, a su vez, se conforma de partículas que pueden descomponerse hasta el infinito. No existe un átomo indivisible y, en consecuencia, no puede existir ninguna esencia, ninguna naturaleza propia (svabhāva).

Ahora bien, si lo que llamamos tiempo es un "devenir", estaríamos aceptando entonces que existen cambios y, por ende, transformación y pérdida de un carácter único y permanente. De ser así, el tiempo - tomado como el conjunto de tres etapas temporales - no puede tener una

2 vijñānam samniviśate saṃskārapratyayam gatau samniviște 'tha vijñāne nāmarūpam niṣicyate.

${ }^{3}$ anapekșya punah siddhir nātītam vidyate tayoḥ pratyutpanno 'nagataś ca tasmāt kālo na vidyate. 
naturaleza fija. Sostener que un objeto existe como tal en los tres tiempos y que, con la transición de un tiempo a otro, ha permanecido igual, sería incurrir en la falsa noción del eternalismo. El budismo, en principio, no puede aceptar esto, ya que una de sus máximas es la impermanencia de las cosas y de toda naturaleza propia. Y es que el término de śünya supone un vaciamiento de esencialismos: "the purport of emptiness is the function of emptiness, not its essence; for it has no essence" (Gómez, "Emptiness and Moral Perfection", 367). En realidad, los tres tiempos son de una sola naturaleza: la carencia de una naturaleza específica (Ramanan, 199).

El tiempo, luego, es impermanente, e impermanentes son también los objetos a través del tiempo. Vasubandhu, exponente de la escuela Yogācāra, opina al respecto que todo perece y que esto lo hace de forma inmediata. De no ser así, el objeto duraría, pero puesto que está sujeto al cambio, debe por necesidad dejar de ser el mismo y perecer, pues sería absurdo que un objeto siga siendo el mismo mientras experimenta cambios periódicamente (Bareau, 2). Así, para Vasubandhu todo objeto no tiene más existencia que de forma inmediata, es decir, durante el instante presente. De manera similar, Bachelard y Roupnel consideran que la realidad no tiene - no puede tener-concreción más que en el instante. La cuestión es que el ser funge como campo de resonancia, es decir, el punto donde hacen eco los instantes. En última instancia, "el recuerdo del pasado y la previsión del porvenir se basan en hábitos" (Bachelard, 48-50), pero, como tales, no existen en tiempo presente, ni son, como también dice Nāgārjuna, realidades que estaban y estarán.

Al mismo tiempo, sin embargo, la vía apofática no necesariamente condujo hacia una nada estática. Como expone Luis Gómez: “Al caracterizar a todo lo existente como cambiante, perecedero, discontinuo, vano, de partida el budismo debió verse obligado a definir un absoluto igualmente fluido, negativo solo en cuanto es inaprehensible" ("Consideraciones en torno al absoluto", 106-107). El meollo del asunto radica en que no se puede decir que el pasado existe, pues entonces su significado perdería sentido, es decir que el pasado perdería su función de preteridad. Lo mismo, se entiende, sucede con el futuro; dado que es el porvenir, está por suceder; no puede existir en el presente: decir será no equivale a es. El ser, por tanto, no existe ni en el pasado ni en el futuro. El budismo Madhyamaka (MMK, II) describe tres puntos u objetos 
de moción, es decir, el espacio que ya se ha cruzado (gata), el que está siendo atravesado (gamyamāna) y el que aun no se cruza (agata). Paralelamente, los agentes o sujetos (gantā) de moción se clasifican en tres tipos: el que se ha movido, el que se mueve y el que aún no se mueve. Estos están a fortiori en correspondencia contemporánea y no pueden mezclarse, es decir que a un agente no se le puede predicar un objeto que no le corresponda: resultaría insensato decir algo así como lo que ha sido atravesado está siendo atravesado por el que aún no se mueve. $\mathrm{O}$, por ejemplo, los caballos llegarán desde ayer. La relación necesaria se rompe y, por ende, toda lógica y coherencia. ${ }^{4}$

En general, el orden en el que se piensan los tres tiempos es casi invariablemente este: pasado, presente y futuro. En la India, paralelo a su elaborado sistema cíclico, se conciben también tres grandes fases de la existencia del universo, representados a su vez en el hinduismo por cada una de las divinidades de la Trimurti, o Trinidad hindú: la creación, la preservación y la destrucción. La preservación, resulta claro, corresponde al tiempo presente, a lo que es, lo que está sucediendo. Con los otros dos periodos resulta un poco más complejo. A primera vista se dirá que siendo que el pasado va cronológicamente al principio, es menester que corresponda con la creación, mientras que la destrucción es futura porque radica en el porvenir, porque implica la aniquilación de lo que es. El problema viene en que la creación - lo nuevo- es también un futuro. Lo nuevo, por necesidad, se sitúa en tiempo futuro; la creación supone crear lo que no hay ahora; es siempre un futuro respecto de un pasado. La destrucción, por otra parte, supone la eliminación de lo que hay o hubo, y de ese modo puede apuntar hacia atrás o hacia delante. Así, el pasado y el futuro parecen hallar cierta equivalencia. Es también por ello que ninguno de los dos existe ahora. $\mathrm{Y}$ a fin de cuentas, ¿quién puede asegurar que existieron o que existirán si no podemos existir sino en el presente?

${ }^{4}$ No obstante, para mantener el juego dialéctico entre filosofía y literatura, vale la pena advertir que escritores como Borges y Cortázar, entre otros, han demostrado que de hecho se puede franquear dicha correspondencia temporal. Pensar por ejemplo en relatos como "La noche boca arriba" y "El jardín de senderos que se bifurcan", por nombrar solo dos. Se dirá, no lo dudo, que en este caso se trata solo de literatura, pero, quién sabe si no hay más de verdad allí que en los tratados científicos. 
El tiempo es tal porque se basa en la acción, en el ser (sea este considerado como real o irreal). Es lo que Bachelard intenta asentar vía la argumentación de Roupnel: el ser es. Para la escuela temprana de los Sarvāstivādins no era tan ajena esta premisa. Para ellos, la temporalidad consistía en la función, porque la unidad del tiempo es la unidad de función (Ramanan, 58). Con esta misma teoría explican ellos los tres tiempos. Puesto que el tiempo y la función son indisolubles, cada periodo de tiempo está basado en su funcionalidad: lo que es, fue o está por ser. La escuela Madhyamaka no está muy lejos de esta perspectiva; Candrakīrti, uno de los principales comentadores de Nāgārjuna, escribió en su Prasannapāda: "Y este estar aquí y allá existe en un momento dado gracias a que se apoya en causas y condiciones. [Pero] esta [afirmación sobre la existencia de los opuestos] es una mera construcción conceptual como la de "corto y largo"” (apud Gómez, "Consideraciones en torno al absoluto", 117).

Importante es destacar que Bachelard - seguramente sin estar consciente de ello - llega a conclusiones similares a las que ya habían llegado siglos antes los budistas y los vedāntins. El sujeto presente aparece en ambos como la conjunción de múltiples agregados:

En el fondo, el individuo no es ya sino una suma de accidentes. [...] tal vez la concepción del individuo como suma integral del ritmo pueda tener una interpretación cada vez menos sustancialista, cada vez más alejada de la materia y cada vez más próxima al pensamiento (Bachelard, 66-67).

Leído en otros términos, esta tesis resulta casi idéntica a la proposición del Advaita Vedānta, la cual afirma que el mundo fenoménico no es sino el reflejo de la Cit, la Conciencia del Brahman. En términos budistas, también el individuo es el conjunto de samskāras o skandhas, es decir, las nociones de forma, las sensaciones, la percepción, la conciencia y las representaciones mentales. El Mülamadhyamaka-kārikā dice:

A partir de la percepción de las naturalezas variantes, todas las entidades carecen de naturaleza propia.

Una entidad sin naturaleza propia no existe 
porque todas las entidades tienen la naturaleza del vacío (śunyatā) (XIII.3). ${ }^{5}$

Bachelard encuentra en la tesis de Roupnel conclusiones bastante similares. Todo cambia, menos el presente momentáneo; solo en ese punto el ser es tal. Compárese la idea arriba citada con estas palabras de Bachelard: "en verdad no existe sino la nada que sea continua". O: "no se debe hablar ni de la unidad ni de identidad del yo fuera de la síntesis realizada por el instante" (36, 65-66).

En todo caso, lo que aquí se ha presentado no es la verdad revelada en Oriente en oposición a la mentira perpetuada por Occidente o viceversa, sino que el tiempo, a final de cuentas, resulta ser menos definible y asequible de lo que parece. La certidumbre que de él tenemos es más bien frágil. Y hay que reconocer al mismo tiempo las similares conclusiones que se han alcanzado tanto en India como en Europa acerca del tiempo. Bachelard, vía Roupnel, coloca solo el instante como entidad real, existente, y como único punto de afirmación del ser. El budismo Madhyamaka, por su parte, también establece la existencia del momento, pero con el objeto de señalar la impermanencia de las cosas. Para ambos, el pasado y el futuro no existen en verdad, pero podríamos decir que para Nāgārjuna incluso el presente carece de existencia absoluta; su existencia es solo relativa porque se desprende del conglomerado de nociones $\mathrm{y}$ falsas concepciones que conforman al individuo:

Si el tiempo existe en virtud de lo existente, ¿cómo puede haber tiempo sin un existente?

Pero ningún existente en verdad existe, ¿cómo puede entonces existir el tiempo? (MMK, XXVI.6) ${ }^{6}$

${ }^{5}$ bhāvānām niḥsvabhāvatvamanyathābhāvadarśanāt asvabhāvo bhāvo nāsti bhāvānām śūnyatā yatah

${ }^{6}$ bhāvam patrītya kālaścetkālo bhāvādṛte kutah na ca kaścana bhāvo 'sti kutaḥ kālo bhavișyati. 
La especie humana parece estar condicionada por un anhelo de duración o de trascendencia, pero dicho anhelo produce una angustia perenne. Como lo expone Luis Gómez: "Esta ansia de duración, connata al hombre, puede, al menos según la doctrina budista, superarse". Sin embargo, continúa Gómez, es importante comprender cómo lograrlo, algo no tan fácil de lograr: "Pero no se supera con el intelecto discursivo, el cual está por naturaleza inmerso en la ilusión de la permanencia" ("Consideraciones en torno al absoluto", 121). La realidad del presente es solo relativa. A partir del Kathāvatthu, Waller (citado por Keith, 300) dedujo que dentro del canon budista, mientras que no se niega la existencia del tiempo trascendente, es claro que se reduce el tiempo al presente momentáneo y que el tiempo es un fenómeno ideal. Así, el pasado y el futuro son de carácter fenoménico, es decir, aparentes, pero no trascendentes. Para Bachelard, en cambio, el instante sí posee una existencia tangible, así como el ser mismo; el ser depende del tiempo y el tiempo real es constituido por el ser. Es la noción de duración lo que resulta intangible e inexistente en última instancia, puesto que ello se desprende de la injerencia del hábito y de la proyección del pensamiento.

Es de todos sabido que Heidegger concibió el ser como un proyecto, como un devenir. Para él, el hecho de que el ser estuviese sujeto al cambio presentaba oportunidades ontológicas más que impermanencia. De ser así, habría que reconocer la dinámica inherente en las actividades, encuentros e interacción entre los seres humanos. Al estar tan estrechamente ligado el ser con el tiempo, ni uno ni otro pueden en realidad ser mostrados; al ser solo se puede serlo. Y el tiempo, también, solo puede ser experimentado. Borges advierte bien que hay realidades que no pueden ser aprehendidas vía el lenguaje: "Todo lenguaje es de índole sucesiva; no es hábil para razonar lo eterno, lo intemporal" (245). El lenguaje no es sino una tentativa de señalar lo que en realidad no puede ser señalado. Pero también, como dice Arnau (106) interpretando a Nāgārjuna: "Que las palabras puedan servir a un fin no tiene por qué significar que tengan una naturaleza propia o que no sean tan vacías como el resto de las cosas".

Para que el tiempo - o la noción temporal del mundo - sea sujeta de experimentación, es preciso la injerencia de otro elemento: el espacio. En esencia, el tiempo no constituye sino la necesidad de medir el movi- 
miento, el transcurrir, y para que este se produzca, es forzoso contar con un espacio. Del mismo modo, no puede haber espacio alguno que no suponga, que no exija la génesis del devenir, del paso del tiempo. El ser, luego, es más que tiempo; el ser es una entidad bipolar, conformada por un elemento temporal y uno espacial, los cuales, en realidad, no pueden ser escindidos.

En medio de esta interacción entre el ente espacial y el ente temporal debemos, no obstante, reconocer factores que pueden modificar el curso de los acontecimientos. Los fenómenos, aunque así lo parezca, no poseen una tendencia irrevocable hacia la linealidad. En efecto, de vez en vez nos hallaremos ante un factor impredecible y definitorio en las trayectorias de los fenómenos. El orden no es una realidad, sino una necesidad del hombre. En verdad, el azar (¿o se trata en realidad de la ley del karma?) posee mucha más injerencia de la que sospechamos. Este aparente orden bajo del cual vivimos es más bien un indómito caos al que tratamos de aprisionar con grilletes, pero ello no es posible. Acaso el problema es que se quiere medir el tiempo pensando en términos lineales, cuando en realidad los fenómenos presentan tendencias recurrentes, es decir, cíclicas. En India se ha tenido más conciencia al respecto. Esto, en efecto, se ha tratado de hacer también en el mundo occidental: a partir de las repeticiones se intenta predecir el curso de un fenómeno dado, pero siempre con la perspectiva progresista que mencioné al principio de esta especulación. El tiempo es caótico, y no puede tener más orden que la ciclicidad: no hay nada en el futuro que no haya estado ya en el pasado, al menos como semilla. Pues quizá lo que se repite no es sino la posibilidad de cambio.

El vacío y el cambio parecen converger: "el mismo concepto de vacuidad, de la absoluta mutabilidad y dependencia de todo lo confeccionado, excluye toda posibilidad de un absoluto aprehensible" (Gómez, "Consideraciones en torno al absoluto", 112). El tiempo está vacío de duración y, paradójicamente, no se puede retener ni contener. Lo que sí resulta seguro es que estos apuntes se detienen aquí y que no han sido sino observaciones preliminares sobre el tema. Tal vez en un momento distinto, otro yo se siente a retomar estas inquietudes. 


\section{REFERENCIAS}

Arnau, Juan, La palabra frente al vacio, México, Fondo de Cultura Económica, 2005.

BACHELARD, Gaston, La intuición del instante [1932], trad. Jorge Ferreiro, México, Fondo de Cultura Económica, 1999.

Bareau, "The Notion of Time", en Essays on Time in Buddhism, H. S. Prasad (ed.), Delhi, Sri Satguru Publications, 1991, 1-12 (Bibliotheca Indo-Buddhica, 78).

Borges, Jorge Luis, "Nueva refutación del tiempo", en Otras inquisiciones, Buenos Aires, Emecé, 1960, 235-257.

Gómez, Luis O., "Emptiness and Moral Perfection", Philosophy East and West, 23, 3, 1973, 361-373.

Gómez, Luis O., "Consideraciones en torno al absoluto de los budistas", Estudios de Asia y África, vol. X, 28:2, 1975, 97-154.

González Reimann, Luis, Tiempo cíclico y eras del mundo en la India, México, El Colegio de México, 1998.

INADA, Kenneth K., Nāgārjuna. A Translation of his 'Mūlamadhyamakakārikā' with an Introductory Essay, Delhi, Sri Satguru Publications, 1993.

Kalupahana, David J., Mūlamadhyamakakārikā of Nāgārjuna. The Philosophy of the Middle Way. Introduction, Sanskrit Text, English Translation and Annotation [1991], Delhi, Moltilal Banarsidas, 1996.

KeIth, "The Doctrine of Reality (The Nature of Time)", en Essays on Time in Buddhism, H. S. Prasad (ed.), Delhi, Sri Satguru Publications, 1991, 299306 (Bibliotheca Indo-Buddhica, 78).

Prasad, H.S., ed., Essays on Time in Buddhism, Delhi, Sri Satguru Publications, 1991 (Bibliotheca Indo-Buddhica, 78).

Ramanan, Venkata, Nāgārjuna's Philosophy as Presented in the Mahā-PrajñāParamitā-Śāstra, Vermont / Tokyo, Charles E. Tuttle Company, 1966. 
